\title{
Joint sub-carrier pairing and resource allocation for cognitive networks with adaptive relaying
}

\author{
Hamza Soury ${ }^{*}$, Faouzi Bader ${ }^{2}$, Musbah Shaat ${ }^{3}$ and Mohamed-Slim Alouini ${ }^{1}$
}

\begin{abstract}
Relayed transmission in a cognitive radio (CR) environment could be used to increase the coverage and capacity of communication system that benefits already from the efficient management of the spectrum developed by CR. Furthermore, there are many types of cooperative communications, including decode-and-forward (DAF) and amplify-and-forward (AAF). In this paper, these techniques are combined in an adaptive mode to benefit from its forwarding advantages; this mode is called adaptive relaying protocol (ARP). Moreover, this work focuses on the joint power allocation in a cognitive radio system in a cooperative mode that operates ARP in multi-carrier mode. The multi-carrier scenario is used in an orthogonal frequency division multiplexing (OFDM) mode, and the problem is formulated to maximize the end-to-end rate by searching the best power allocation at the transmitters. This work includes, besides the ARP model, a sub-carrier pairing strategy that allows the relays to switch to the best sub-carrier pairs to increase the throughput. The optimization problem is formulated and solved under the interference and power budget constraints using the sub-gradient algorithm. The simulation results confirm the efficiency of the proposed adaptive relaying protocol in comparison to other relaying techniques. The results show also the consequence of the choice of the pairing strategy.
\end{abstract}

Keywords: Cognitive radio; Cooperative communication; Amplify-and-forward; Decode-and-forward; Power allocation; Pairing; Adaptive relaying protocol

\section{Introduction}

The growth of technology has affected directly modern communication systems. This expansion can be observed when a comparison is made between the earlier systems with some bits per second as a communication rate and the $300 \mathrm{Mbps}$ already considered in the long-term evolution (LTE) wireless communication systems. The growth of the data rate in wireless standards and services was accompanied by a rise in applications and costumers which implies a strong increase in the demand for the limited frequency spectrum. This means that the actual available spectrum resource may not be able to respond to the emerging and future technology demands.

In current systems, the frequency allocation, the type of service, the maximum transmission powers, and the time duration of the licenses are managed by governmental

\footnotetext{
*Correspondence: soury.hamza@kaust.edu.sa

${ }^{1}$ Computer, Electrical, and Mathematical Sciences and Engineering (CEMSE) Division, King Abdullah University of Science and Technology (KAUST), Thuwal, Makkah Province, 23955-6900 Kingdom of Saudi Arabia

Full list of author information is available at the end of the article
}

agencies, which apply the 'command-and-control' allocation model by assigning a fixed frequency block for each communication service. This scheme is statistic and inflexible in spectrum management which leads, as shown by practical measurements, to inefficient use of the provided spectrum because licensed users are not necessarily using the allocated portion of spectrum at all times or over all the spatial locations, and at the same time prevent other users from accessing the unused spectrum.

Cognitive radio (CR) can manage the spectrum utilization by detecting spectrum holes and avoiding the occupied spectrum using the available part of the spectrum. In fact, the spectrum utilization can be improved by allowing the secondary users (SUs) to use the vacant channels left by the licensed users (PUs) [1]. Such systems have to distribute their limited resources among the SUs in order to maximize the capacity without causing harmful interference to the PUs (see, e.g., $[2,3]$ ). Since OFDM is widely used in various wireless system and shows a high spectral efficiency and flexibility, it is often recommended for cognitive radio systems [4].

\section{至 Springer}

(c) 2013 Soury et al.: licensee Springer. This is an Open Access article distributed under the terms of the Creative Commons Attribution License (http://creativecommons.org/licenses/by/2.0), which permits unrestricted use, distribution, and reproduction in any medium, provided the original work is properly cited. 
To increase coverage and achievable capacity of the communication system, relays $(\mathrm{R})$ are used to transfer the information from the cognitive source (CS) to the destination (D) when the direct link is not available [5] (in some cases even if a direct link exists, the relays are used to improve the performance of the communication systems). The resource allocation problem for the non-cognitive OFDM based relay system has been widely studied [6,7]. In [8], a cooperative scheme with decode-and-forward technique is combined with the cognitive radio to produce a communication system with high performance and higher coverage area. Note that in cognitive cooperative communication systems, both transmitters, namely source and relay, have to be aware about the interference threshold tolerated by the PU.

In cooperative communication systems, the most known relaying techniques are amplify-and-forward (AAF) [9] and decode-and-forward (DAF) [10]. In the AAF case, the $R$ amplifies the received signal from the source (S) by some fixed factor, then forward it to D. However, the relay using the DAF strategy decodes 'perfectly' the received signal from $S$ and then encodes it again (with the same code known by $S$ and D) and forwards it to D. Note that these procedures are done at each sub-carrier. The disadvantages of these two techniques of relaying come with the fact that: (1) AAF relaying can amplify the noise coming form the (S-R) link, which degrades the signal quality, and (2) DAF relaying causes a propagation of error in case of uncorrect decoding of the information symbols.

Adaptive relaying or Adaptive Relaying Protocol (ARP), as named in [6], is one of the proposed solutions that benefits from the advantages of DAF and AAF, and aims to minimize the disadvantages of these two relaying techniques. In $[7,11]$, the relay can execute AAF and DAF, and there is a technique based on the signal-to-noise-ratio (SNR) which triggers the switching between the AAF and DAF strategies. It assumes that at high SNR (for an SNR above some SNR thresholds), the relay can decode perfectly, so it is better to operate with DAF; for low SNRs (below a certain threshold), when it is harder to decode correctly, it is preferable to use the AAF to avoid propagation errors.

The objective of this paper is to provide an efficient procedure to integrate the adaptive relaying technique in a CR-based environment for a joint optimization of the choice of pairing strategy and the power allocation at the transmitters ( $\mathrm{S}$ and $\mathrm{R}$ ) to reach high capacity, without causing harmful interference to the primary user. The proposed solution goes through an algorithm based on the dual problem and sub-gradient method [12-14]. For simplicity, we begin by selecting the sub-carrier and assume that the relay uses the same sub-carrier for receiving (from S) and for transmission (to D). We also consider other type of pairing selection, like random sub-carrier selection and optimal sub-carrier selection from S-R to $\mathrm{R}-\mathrm{D}$ and compare the performances of these different schemes.

\subsection{Related work}

Many works were done to solve the power allocation problem for the cooperative cognitive radio communication systems. In [15], Zou et al. study the spectrum sensing of the cognitive system using a cooperative relay cognitive radio system. Their work focuses on the trade off between the spectrum sensing and SU transmission. The use of relays is presented also in [16], where the authors study the outage probability of the SU when DAF is used. In [17], a cooperative scheme has been used with DAF relaying. [17] deals also with a multiple relay systems and relay selection strategy. The AAF and DAF are both used in [18], where the system decides to use one of these schemes according to the known CSI. If the relay operates in AAF mode, it will amplify the received signal. However, for the DAF mode, if decoding is unsuccessful, the relay will remain silent. Otherwise, the relay re-encodes the decoded data and transmits it to the destination. As we will explain later, we use a different scheme in our work focus on the cognitive context, and this yields another optimization problem. In cite [19], a similar scheme was presented using the sub-carrier pairing technique and AAF forwarding strategy only. On the other hand, similar scheme were presented in [20-22] (with relay selection and sub-carrier pairing); these works focused on power allocation of a relaying system in cognitive radio scenario using only the DAF technique without considering the AAF technique. The contribution of the present work is the use of an adaptive scheme of relaying based on switching between both techniques, i.e., AAF and DAF.

\subsection{Summary of contribution}

This paper proposes a new adaptive relaying protocol based in the AAF and DAF modes. In this protocol, the relays are able to perform the AAF and the DAF according to the capability of the relay to decode successfully the signal. Note that the decision of switching, between both modes, is based not only on the channel information but also on the received SNR which will add more complexity on the optimization problem to maximize the total rate subject to power and interference constraints; the ARP is described in [6], but it is not used for cognitive radio as it is in this paper. Also, the optimization problem to maximize the total rate is missing in [6]. This paper deals also, in a second part, with the sub-carrier pairing problem using the ARP model which differs from the multi-relays especially in the interference computations. Finally, this paper uses the dual problem and sub-gradient algorithm to solve numerically the optimization problem. 


\subsection{Outline of the paper}

The remainder of the paper is organized as follows. In Section 2, we present the system model and the matching sub-carrier problem with a proposed algorithm. The proposed solution is illustrated by some selected numerical results to compare the performance over the different types of relaying (AAF, DAF, and ARP). In Section 3, we investigate the pairing problem by including the pairing parameters within the optimization problem studied in Section 2. More specifically, the same algorithm is used with some modifications to find the best sub-carrier distribution, and we end the section with some simulation results showing the difference between the pairing techniques used in this work. Finally, we conclude this work in Section 4, with a summary of the main results.

\section{Near-optimal algorithm for sub-carrier matching scheme}

In this section, we focus on the simple case in which the power allocation of a cognitive system with one relay system using a matching pairing strategy is adopted. In particular, we assume that the relay forwards the signal over the same received sub-carrier and study the difference in performance between the three types of relaying schemes introduced in the previous section.

\subsection{System model}

In this work, an OFDM-based relay CR system is considered. The CR relay system coexists with the primary system in the same geographical location. We assume that there is no direct link between the CS and the D, so S tries to communicate with $\mathrm{D}$ through the relay (see Figure 1). The frequency spectrum of the CR system is divided into $N$ sub-carriers, each having a $\Delta f$ bandwidth. We assume that the CR system can transmit through the unused PU band without exceeding the maximum interference power $I_{\text {th }}$ that can be tolerated by PU. The relay is assumed to be half-duplex, so receiving and forwarding at two different time slots. In the first time slot, $\mathrm{S}$ transmits to $\mathrm{R}$, while in the second time slot, $\mathrm{R}$ forwards the signal to $\mathrm{D}$ with the ARP technique. It has been assumed that we have one relay which can work with different channels. Note that the relay can forward the data using two techniques (DAF and AAF) by switching between them, as presented in Figure 2. The calculation of the mutual interference between PU, SU, and the relay is presented in the next part.

\subsection{Interference analysis}

The mutual interference introduced to PU by the $i$ th subcarrier in OFDM systems is presented in [23]. Assume that $\Phi_{i}$ is the power spectrum density (PSD) of the $i$ th sub-carrier. The form of the PSD depends directly on the multi-carrier wave form technique. In our case, when an OFDM-based system is used, the PSD at the $i$ th subcarrier band can be written as

$$
\Phi_{i}(f)=P_{i} T_{\mathrm{s}}\left(\frac{\sin \pi f T_{\mathrm{s}}}{\pi f T_{\mathrm{s}}}\right)^{2},
$$

where $P_{i}$ is the total transmit power emitted by the $i$ th sub-carrier, and $T_{\mathrm{s}}$ is the symbol duration. Hence, the mutual interference introduced by the $i$ th sub-carrier to PU, $I_{i}\left(d_{i}, P_{i}\right)$, can be found by integrating the PSD of the $i$ th sub-carrier over the PU band, $B$, and can be obtained using the following expression [8]:

$$
I_{i}\left(d_{i}, P_{i}\right)=\int_{d_{i}-B / 2}^{d_{i}+B / 2} G_{i} \Phi_{i}(f) d f \triangleq P_{i} \Omega^{i},
$$

where $d_{i}$ and $G_{i}$ denote the spectral distance and the channel gain, respectively, between the $i$ th sub-carrier and the PU band, while $\Omega^{i}$ is the interference factor of the $i$ th subcarrier to the PU band [24] ${ }^{\mathrm{a}}$. Note that (2) expresses the interference in terms of the total transmit power $P_{i}$ of the $i$ th sub-carrier linearly, which will be used to solve the optimization problem in the next subsections.

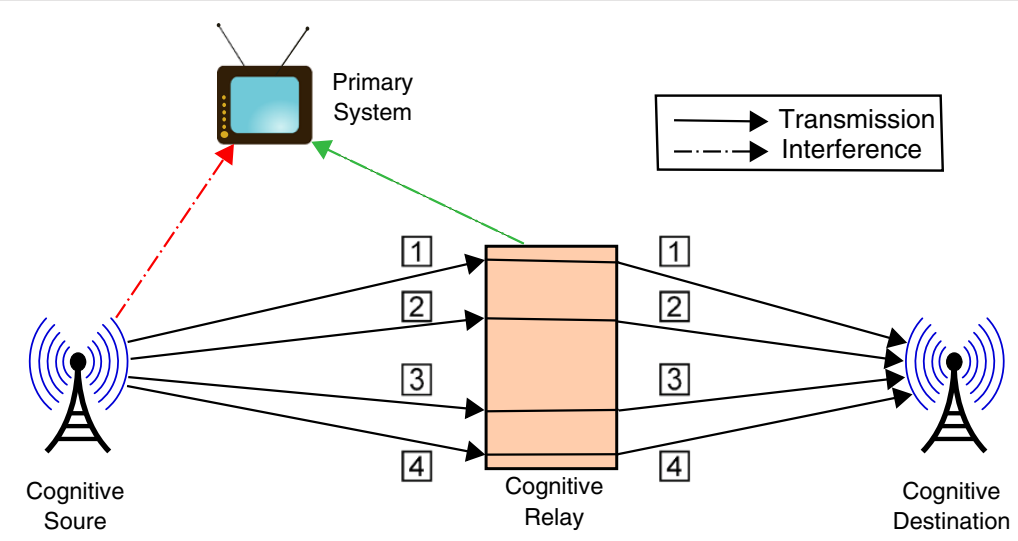

Figure 1 Illustration of a cooperative relay cognitive radio network with $N=4$. 


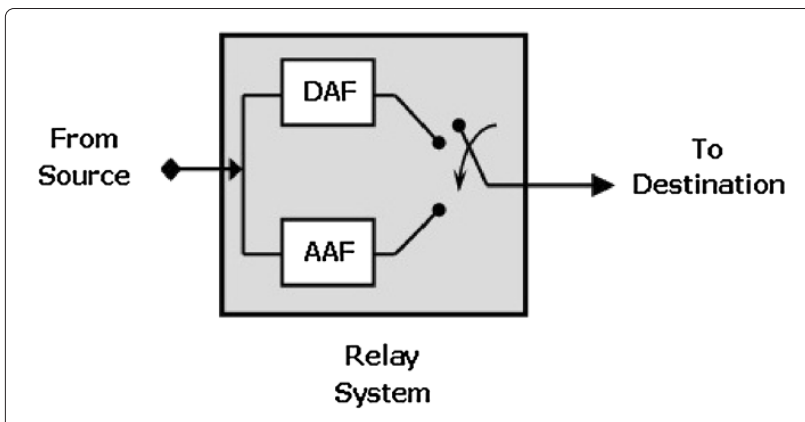

Figure 2 Block diagram for the structure of an adaptive relay.

By the same analysis, the interference power introduced by PU signal into the band of the $i$ th sub-carrier is expressed as [8]

$$
J_{i}=\int_{d_{i}-\Delta f / 2}^{d_{i}+\Delta f / 2} Y_{i} \Psi(f) d f
$$

where $\Psi(f)$ is the PSD of PU signal, and $Y_{i}$ is the channel gain between the $i$ th sub-carrier and the PU signal. By completing the interference analysis of the different agents of the cognitive system, we can formulate the optimization problem before proceeding to the solution.

\subsection{Capacity analysis and problem formulation}

Let us first define the variables of the problem. Let $\left(P_{\mathrm{SR}}^{i} ; P_{\mathrm{RD}}^{i}\right)$ be the power transmitted over the $i$ th subcarrier in the (S-R;R-D) link. The $i$ th sub-carrier channel gain over the (S-R;R-D) link is given by $\left(H_{\mathrm{SR}}^{i} ; H_{\mathrm{RD}}^{i}\right)$. Finally, the noise variance is assigned by $\sigma_{i}^{2}=\sigma_{\text {AWGN }}^{2}+J_{i}$, where $\sigma_{\text {AWGN }}^{2}$ is the variance of the additive white Gaussian noise (AWGN), and $J_{i}$ is the interference introduced by the PU signal into the $i$ th sub-carrier which is evaluated using (3). This interference can be modeled as an AWGN as described in [2]. To make the analysis more clear, the noise variance $\sigma^{2}$ is assumed to be the same for all sub-carriers and both time slots.

\subsubsection{Processing during the first time slot}

Let $x_{\mathrm{s}, i}$ be the transmitted signal from $\mathrm{S}$ over the $i$ th channel. The received signal at the relay $\mathrm{R}$ over the $i$ th sub-carrier in the first time slot is given by

$$
y_{\mathrm{SR}}^{i}=\sqrt{H_{\mathrm{SR}}^{i} P_{\mathrm{SR}}^{i}} x_{\mathrm{s}, i}+n_{\mathrm{SR}}^{i}
$$

where $n_{\mathrm{SR}}^{i}$ is the noise between $\mathrm{S}$ and $\mathrm{R}$ with a variance $\sigma_{\mathrm{SR}, i}^{2}=\sigma^{2}$, and $i=\{1,2, \ldots N\}$ denote the $i$ th sub-carrier.
According to the Shannon capacity formula, the transmission rate of the $i$ th sub-carrier between the source and the relay $R_{1, i}$ can be calculated as

$$
R_{1, i}=\frac{1}{2} \log _{2}\left(1+\frac{P_{\mathrm{SR}}^{i} H_{\mathrm{SR}}^{i}}{\sigma^{2}}\right) .
$$

As it has been mentioned above, we should limit the interference caused by the CS to the PU, which gives us the following interference constraint [8]:

$$
\sum_{i=1}^{N} P_{\mathrm{SR}}^{i} \Omega_{\mathrm{SP}}^{i} \leq I_{t h}
$$

where $\Omega_{\mathrm{SP}}^{i}$ denotes the interference factor of the $i$ th subcarrier to the PU band.

\subsubsection{Capacity in the second time slot}

In the second time slot, the relay decodes and re-encodes or amplifies the signal over the $i$ th channel depending on the received SNR, then forwards it to the destination. This means that the transmit signal from the relay over the $i$ th channel is

$$
x_{\mathrm{RD}}^{i}= \begin{cases}\sqrt{P_{\mathrm{RD}}^{i}} \cdot x_{\mathrm{S}, i} & \text { for } P_{\mathrm{SR}}^{i} \gamma_{\mathrm{SR}}^{i} \geq \gamma_{t h} \\ \beta_{i} \sqrt{P_{\mathrm{RD}}^{i}} \cdot y_{\mathrm{SR}}^{i} & \text { for } P_{\mathrm{SR}}^{i} \gamma_{\mathrm{SR}}^{i}<\gamma_{t h},\end{cases}
$$

where $P_{\mathrm{SR}}^{i} \gamma_{\mathrm{SR}}^{i}=\frac{P_{\mathrm{SR}}^{i} H_{\mathrm{SR}}^{i}}{\sigma^{2}}$ is the received SNR via the source-relay link, and $\gamma_{t h}$ is the threshold SNR to ensure successful decoding. We assume that we have successful decoding when $P_{\mathrm{SR}}^{i} \gamma_{\mathrm{SR}}^{i}$ is above $\gamma_{t h}$. In (7), $\beta_{i}$ is an amplification factor used by the relay to amplify the signal using the AAF mode. The choice of $\beta_{i}$ should assure the normalization of the total transmit power to the same value with all the AAF channels. It is defined in ([25], Eq. (9)) as

$$
\beta[i]=\frac{1}{\sigma \sqrt{P_{\mathrm{SR}}^{i} \gamma_{\mathrm{SR}}^{i}+1}} .
$$

At the destination, the received signal over the $i$ th channel can be written as

$$
y_{\mathrm{RD}}^{i}=\sqrt{H_{\mathrm{RD}}^{i}} x_{\mathrm{RD}}^{i}+n_{\mathrm{RD}}^{i}
$$

Let us define two variables: $\gamma_{\mathrm{SR}}^{i}=\frac{H_{\mathrm{SR}}^{i}}{\sigma^{2}}$ and $\gamma_{\mathrm{RD}}^{i}=\frac{H_{\mathrm{RD}}^{i}}{\sigma^{2}}$. Using (7) and (9), we derive the expression of the total SNR delivered via the $i$ th channel as

$$
\gamma_{\mathrm{ARP}}^{i}= \begin{cases}P_{\mathrm{RD}}^{i} \gamma_{\mathrm{RD}}^{i} & \text { if } P_{\mathrm{SR}}^{i} \gamma_{\mathrm{SR}}^{i} \geq \gamma_{t h} \\ \gamma_{\mathrm{AF}}^{i} & \text { otherwise }\end{cases}
$$


where $\gamma_{\mathrm{AF}}^{i}$ is the SNR for the set of channels that work on amplify-and-forward, and it is given by

$$
\begin{aligned}
\gamma_{\mathrm{AF}}^{i} & =\frac{\mathbb{E}\left[y_{\mathrm{RD}, \mathrm{AAF}}^{i}\right]^{2}}{\mathbb{E}\left[\left(y_{\mathrm{RD}, \mathrm{AAF}}^{i}\right)^{2}\right]-\mathbb{E}\left[y_{\mathrm{RD}, \mathrm{AAF}}^{i}\right]^{2}} \\
& =\frac{P_{\mathrm{SR}}^{i} \gamma_{\mathrm{SR}}^{i} P_{\mathrm{RD}}^{i} \gamma_{\mathrm{RD}}^{i}}{P_{\mathrm{RD}}^{i} \gamma_{\mathrm{RD}}+P_{\mathrm{SR}}^{i} \gamma_{\mathrm{SR}}^{i}+1},
\end{aligned}
$$

where $\mathbb{E}[Y]$ denotes the expected value of the random variable $Y$.

Back to the Shannon capacity formula, we calculate the rate of the channel in the second time slot for the two cases as

$$
\left\{\begin{array}{c}
R_{2, \mathrm{DAF}, i}=\frac{1}{2} \log _{2}\left(1+\frac{P_{\mathrm{RD}}^{i} H_{\mathrm{RD}}^{i}}{\sigma^{2}}\right) \text { for the DAF case } \\
R_{2, \mathrm{AAF}, i}=\frac{1}{2} \log _{2}\left(1+\frac{P_{\mathrm{RD}}^{i} \gamma_{\mathrm{RD}}^{i} P_{\mathrm{S}}^{i} \gamma_{\mathrm{SR}}^{i}}{P_{\mathrm{RD}}^{i} \gamma_{\mathrm{RD}}^{i}+P_{\mathrm{SR}}^{i} \gamma_{\mathrm{SR}}^{i}+1}\right) \\
\text { for the AAF case. }
\end{array}\right.
$$

Note that $R_{2, \mathrm{AAF}, i}$ is not jointly concave in $P_{\mathrm{RD}}^{i}$ and $P_{\mathrm{SR}}^{i}$. To make the analysis simpler, we adopt the following approximation:

$$
R_{2, \mathrm{AAF}, i} \approx \frac{1}{2} \log _{2}\left(1+\frac{P_{\mathrm{RD}}^{i} \gamma_{\mathrm{RD}}^{i} P_{\mathrm{SR}}^{i} \gamma_{\mathrm{SR}}^{i}}{P_{\mathrm{RD}}^{i} \gamma_{\mathrm{RD}}^{i}+P_{\mathrm{SR}}^{i} \gamma_{\mathrm{SR}}^{i}}\right) .
$$

This approximation is used in [26], and it is based on the assumption that the system has a high SNR for the amplified signal between the relay and the destination. It is proved in [27] that this approximation is also accurate even in the moderate-low SNR regime.

To make things more clear, a new binary variable $\alpha_{i}$ is defined in a way that it takes the values ' 0 ' or ' 1 ' to indicate if the relay uses the DAF case $\left(\alpha_{i}=0\right)$ or the AAF case $\left(\alpha_{i}=1\right)$. We denote also by $\mathcal{A}$ the set of index of channels that work on AAF, and $\mathcal{D}$ as the set of index of channels that work on DAF

$$
\mathcal{A}=\left\{i, \alpha_{i}=1\right\} ; \mathcal{D}=\left\{i, \alpha_{i}=0\right\} .
$$

As in the DAF case, we compute the interference caused by the relay to the PU for the AAF case. Using the interference analysis done above and the expression given in ([28], Eq. (17-18)), we get the following interference constraint in the second time slot $(R \longrightarrow D)$ :

$$
\sum_{i \in \mathcal{A}} P_{\mathrm{RD}}^{i} \Omega_{\mathrm{RP}}^{i}+\sum_{i \in \mathcal{D}} P_{\mathrm{RD}}^{i} \Omega_{\mathrm{RP}}^{i} \leq I_{t h} .
$$

\subsubsection{Total capacity}

It is clear that the capacity has different expression in each time slot and forwarding technique. Therefore, we need to find a unified expression that will be used as the objective function of the upcoming optimization problem. In fact, the achievable rate in each sub-carrier is the minimum rate between both time slots. Thus, the transmission rate is given by

$$
R_{i}=\alpha_{i} \min \left\{R_{1, i}, R_{2, \mathrm{DAF}, i}\right\}+\left(1-\alpha_{i}\right) \min \left\{R_{1, i}, R_{2, \mathrm{AAF}, i}\right\} .
$$

The maximum capacity is achievable when the rate in the first time slot is equal to the rate in the second time slot for every sub-carrier if it is possible. Thus, from (16), we should have the following capacity relation for the $i$ th sub-carrier to achieve maximum rate:

$$
R_{1, i}=\left\{\begin{array}{l}
R_{2, \mathrm{DAF}, i} \text { for } i \in \mathcal{D} \\
R_{2, \mathrm{AAF}, i} \text { for } i \in \mathcal{A} .
\end{array}\right.
$$

Hence, we get two cases. For the DAF case, the equality is achievable by assembling (17), (5), and (12) to derive the following relation between the transmission powers:

$$
P_{\mathrm{RD}}^{i}=\frac{P_{\mathrm{SR}}^{i} H_{\mathrm{SR}}^{i}}{H_{\mathrm{RD}}^{i}} \quad \text { for } i \in \mathcal{D} .
$$

However, if we look to the formula of the rates in the AAF case (12), we can see that the rate in the second time slot is always less than the rate in the first time slot and cannot reach it. In fact, we have

$$
\frac{P_{\mathrm{RD}}^{i} \gamma_{\mathrm{RD}}^{i} P_{\mathrm{SR}}^{i} \gamma_{\mathrm{SR}}^{i}}{P_{\mathrm{RD}}^{i} \gamma_{\mathrm{RD}}^{i}+P_{\mathrm{SR}}^{i} \gamma_{\mathrm{SR}}^{i}}<\frac{P_{\mathrm{SR}}^{i} H_{\mathrm{SR}}^{i}}{\sigma^{2}} \text { for } i \in \mathcal{A} .
$$

This means that the achievable rate of these channels is equal to the rate in the second time slot. According to these derivations, we find the total expression of the rate in our model as

$$
\begin{aligned}
R= & \sum_{i \in \mathcal{A}} \frac{1}{2} \log _{2}\left(1+\frac{P_{\mathrm{RD}}^{i} \gamma_{\mathrm{RD}}^{i} P_{\mathrm{S}}^{i} \gamma_{\mathrm{SR}}^{i}}{P_{\mathrm{RD}}^{i} \gamma_{\mathrm{RD}}^{i}+P_{\mathrm{SR}}^{i} \gamma_{\mathrm{SR}}^{i}}\right) \\
& +\sum_{i \in \mathcal{D}} \frac{1}{2} \log _{2}\left(1+P_{\mathrm{SR}}^{i} \gamma_{\mathrm{SR}}^{i}\right) .
\end{aligned}
$$

\subsubsection{Optimization problem of the sub-carrier matching technique}

Our objective is to maximize the total capacity of the CR system while the interference introduced to the primary user is below the tolerated threshold. Therefore, the optimization problem can be formulated as follows:

$\max _{P_{\mathrm{SR}}^{i}, P_{\mathrm{RD}}^{i}} \sum_{i=1}^{N} R_{i}$

Subject to

- (Interference at first time slot)

$\sum_{i=1}^{N} P_{\mathrm{SR}}^{i} \Omega_{\mathrm{SP}}^{i} \leq I_{\text {th }}$

- (Interference at second time slot)

$$
\begin{aligned}
& \sum_{i \in \mathcal{A}} P_{\mathrm{RD}}^{i} \Omega_{\mathrm{RP}}^{i}+\sum_{i \in \mathcal{D}} P_{\mathrm{RD}}^{i} \Omega_{\mathrm{RP}}^{i} \leq I_{t h} \\
& P_{\mathrm{SR}}^{i} \geq 0 ; \quad P_{\mathrm{RD}}^{i} \geq 0 .
\end{aligned}
$$


In this problem, the power constraints at each transmitter ( $\mathrm{S}$ and $\mathrm{R}$ ) are missed. However, when we take a look at the interference constraints, we note that the power constraint is defined indirectly. Moreover, we use the identity $\Omega_{\mathrm{SP}}^{i} \geq \min _{j} \Omega_{\mathrm{SP}}^{j}$ to ensure the following inequality $\sum_{i=1}^{N} P_{\mathrm{SR}}^{i} \leq \frac{I_{t h}}{\min _{j}^{j} \Omega_{\mathrm{SP}}^{j}}$. Thus, the interference constraint implies, indirectly, a power constraint in the two time slots; even if the resulting interference is too small, the problem remains approximately the same without significant change. This analysis can help us in this chapter because the problem is relatively simple and is not a mixed integer programming problem, so the power constraint can be omitted. However, in the next section, the problem is more complex, and we should define the power constraints at the transmitters to avoid non-convergence of the algorithm.

We assume that all fading gains are perfectly known. The channel gains between the CR system parts $(S, R$, and D) can be obtained by channel estimation techniques; the channel gains between the CR system and the PU can be obtained by estimating the received signal power from the primary terminal when it transmits [29]. At the end of this part and by assembling the previous equations and relations, we can re-write the optimization problem given in (21) as

$$
\begin{gathered}
\max _{P_{\mathrm{SR}}^{i}, P_{\mathrm{RD}}^{i}} \frac{1}{2} \sum_{i \in \mathcal{A}} \log _{2}\left(1+\frac{P_{\mathrm{RD}}^{i} \gamma_{\mathrm{RD}}^{i} P_{\mathrm{SR}}^{i} \gamma_{\mathrm{SR}}^{i}}{P_{\mathrm{RD}}^{i} \gamma_{\mathrm{RD}}^{i}+P_{\mathrm{SR}}^{i} \gamma_{\mathrm{SR}}^{i}}\right) \\
+\frac{1}{2} \sum_{i \in \mathcal{D}} \log _{2}\left(1+P_{\mathrm{SR}}^{i} \gamma_{\mathrm{SR}}^{i}\right) \\
\text { s.t. } \\
\sum_{i=1}^{N} P_{\mathrm{SR}}^{i} \Omega_{\mathrm{SP}}^{* i} \leq I_{t h} ; \\
\sum_{i \in \mathcal{A}} P_{\mathrm{RD}}^{i} \Omega_{\mathrm{RP}}^{i}+\sum_{i \in \mathcal{D}} \frac{P_{\mathrm{SR}}^{i} H_{\mathrm{SR}}^{i}}{H_{\mathrm{RD}}^{i}} \Omega_{\mathrm{RP}}^{i} \leq I_{t h} ; \\
P_{\mathrm{SR}}^{i} \geq 0 ; \\
P_{\mathrm{RD}}^{i} \geq 0 .
\end{gathered}
$$

Under the previous assumption of perfect knowledge of the channel coefficient and the noise variance, the problem is a convex optimization problem with the parameter $P_{\mathrm{RD}}^{i}$ and $P_{\mathrm{SR}}^{i}$. In the next part, we solve this problem using the Lagrangian method and the Karush-Kuhn-Tucker (KKT) conditions. Moreover, using the fact that the problem is convex, the dual solution and the primal solution are the same, so the problem can be solved using the dual formulation.

\subsection{Solution}

For simplicity reasons and for making the mathematical notation easy to follow, we denote the following: $P_{\mathrm{SR}}^{i}$ by $P_{1}^{i}$, $P_{\mathrm{RD}}^{i}$ by $P_{2}^{i}, \gamma_{\mathrm{SR}}^{i}$ by $\gamma_{1}^{i}, \gamma_{\mathrm{RD}}^{i}$ by $\gamma_{2}^{i}, \Omega_{\mathrm{SP}}^{i}$ by $\Omega_{1}^{i}$, and $\Omega_{\mathrm{RP}}^{i}$ by $\Omega_{2}^{i}$.

\subsubsection{Dual problem}

The Lagrangian function with Lagrangian multipliers $\lambda, \mu$ can be written as presented in (23).

$$
\begin{aligned}
\mathcal{L}= & \frac{1}{2} \sum_{i=1}^{N}\left[\alpha_{i} \log _{2}\left(1+\frac{P_{1}^{i} \gamma_{1}^{i} P_{2}^{i} \gamma_{2}^{i}}{P_{1}^{i} \gamma_{1}^{i}+P_{2}^{i} \gamma_{2}^{i}}\right)+\left(1-\alpha_{i}\right) \log _{2}\left(1+P_{1}^{i} \gamma_{1}^{i}\right)\right] \\
& +\mu\left(I_{t h}-\sum_{i=1}^{N} P_{1}^{i} \Omega_{1}^{i}\right)+\lambda\left(I_{t h}-\sum_{i=1}^{N} \alpha_{i} P_{2}^{i} \Omega_{2}^{i}\right. \\
& \left.+\left(1-\alpha_{i}\right) \frac{P_{1}^{i} H_{\mathrm{SR}}^{i}}{H_{\mathrm{RD}}^{i}} \Omega_{2}^{i}\right) .
\end{aligned}
$$

Note that we substitute $\mathcal{A}$ and $\mathcal{D}$ by their definition, and we include $\alpha_{i}$ in the Lagrangian to simplify the computation. We develop the Lagrangian to get the following expression:

$$
\begin{aligned}
\mathcal{L}= & \sum_{i=1}^{N} \alpha_{i}\left[\frac{1}{2} \log _{2}\left(1+\frac{P_{1}^{i} \gamma_{1}^{i} P_{2}^{i} \gamma_{2}^{i}}{P_{1}^{i} \gamma_{1}^{i}+P_{2}^{i} \gamma_{2}^{i}}\right)-\lambda P_{2}^{i} \Omega_{2}^{i}-\mu P_{1}^{i} \Omega_{1}^{i}\right] \\
& +\left(1-\alpha_{i}\right)\left[\frac{1}{2} \log _{2}\left(1+P_{1}^{i} \gamma_{1}^{i}\right)-\lambda \frac{P_{1}^{i} H_{\mathrm{SR}}^{i}}{H_{\mathrm{RD}}^{i}} \Omega_{2}^{i}-\mu P_{1}^{i} \Omega_{1}^{i}\right] \\
& +(\lambda+\mu) I_{t h} .
\end{aligned}
$$

Now, we solve this problem using the dual approach. But first, let us define the dual problem and the dual function as

$$
\min _{\mu \geq 0, \lambda \geq 0} g(\mu, \lambda)
$$

where

$$
g(\mu, \lambda) \triangleq \max _{P_{1}^{i} \geq 0, P_{2}^{i} \geq 0, \alpha_{i}} \mathcal{L} .
$$

From (24) and for a given set of $\alpha_{i}$, the problem can be divided into $N$ independent problems. Thus, we divide the dual function (the Lagrangian) into $N$ dual functions (Lagrangian), such that $g_{i}\left(\mathcal{L}_{i}\right)$. For each sub-carrier $i$, for given $\lambda$ and $\mu$, and according to the value of $\alpha_{i}$ (which can take two values, 0 or 1 ), it appears that there are two cases:

- Case $\alpha_{i}=1$. In this case, the relay is working on AAF for the ith sub-carrier. As we have in (23), only the terms related to the AAF approach. Hence, the dual function can be simplified as follows:

$$
\begin{aligned}
g_{i}(\mu, \lambda)= & \max _{P_{1}^{i}, P_{2}^{i} \geq 0} \mathcal{L}_{i} \\
=\max _{P_{1}^{i}, P_{2}^{i} \geq 0} & \frac{1}{2} \log _{2}\left(1+\frac{P_{1}^{i} \gamma_{1}^{i} P_{2}^{i} \gamma_{2}^{i}}{P_{1}^{i} \gamma_{1}^{i}+P_{2}^{i} \gamma_{2}^{i}}\right) \\
& \quad-\lambda P_{2}^{i} \Omega_{2}^{i}-\mu P_{1}^{i} \Omega_{1}^{i} .
\end{aligned}
$$


The maximum of $\mathcal{L}_{i}$ can be found by searching the partial derivative of $\mathcal{L}_{i}$ subject to $P_{1}^{i}$ and $P_{2}^{i}$ which leads to

$$
\frac{\partial \mathcal{L}_{1}}{\partial P_{1}^{i}}=\frac{\left(P_{2}^{i}\right)^{2}\left(\gamma_{2}^{i}\right)^{2} \gamma_{1}^{i}}{\left(P_{2}^{i} \gamma_{2}^{i}+P_{1}^{i} \gamma_{1}^{i}\right)\left(P_{2}^{i} \gamma_{2}^{i}+P_{1}^{i} \gamma_{1}^{i}+P_{2}^{i} \gamma_{2}^{i} P_{1}^{i} \gamma_{1}^{i}\right)}-\mu \Omega_{1}^{i}
$$

$$
\frac{\partial \mathcal{L}_{1}}{\partial P_{2}^{i}}=\frac{\left(P_{1}^{i}\right)^{2}\left(\gamma_{1}^{i}\right)^{2} \gamma_{2}^{i}}{\left(P_{2}^{i} \gamma_{2}^{i}+P_{1}^{i} \gamma_{1}^{i}\right)\left(P_{2}^{i} \gamma_{2}^{i}+P_{1}^{i} \gamma_{1}^{i}+P_{2}^{i} \gamma_{2}^{i} P_{1}^{i} \gamma_{1}^{i}\right)}-\lambda \Omega_{2}^{i}
$$

We then equal both (27) and (28) to zero. The solution of these equations leads to $P_{1}^{i *}=c_{i} P_{2}^{i *}$, where $c_{i}=\sqrt{\frac{\gamma_{2}^{i} \lambda \Omega_{2}^{i}}{\gamma_{1}^{i} \mu \Omega_{1}^{i}}}$. Thus, the new value of $P_{2}^{i}$ is

$$
P_{2}^{i *}=\left[\frac{\gamma_{2}^{i}}{\mu c_{i} \Omega_{1}^{i}\left(\gamma_{2}^{i}+c_{i} \gamma_{1}^{i}\right)}-\frac{1}{c_{i} \gamma_{1}^{i}}-\frac{1}{\gamma_{2}^{i}}\right]^{+},
$$

where $[x]^{+}=\max (0, x)$.

- Case $\alpha_{i}=0$. For this case, the relay switches to the DAF technique at the ith sub-carrier having $i \in \mathcal{D}$. The problem of the DAF relaying has been solved in [8]. We just have to know the value of $P_{1}^{i *}$ which can be obtained from the following relation $P_{2}^{i *}=\frac{P_{1}^{i *} H_{\mathrm{SR}}^{i}}{H_{\mathrm{RD}}^{i}}$ The solution is found to be given, in this case, by the following expression:

$$
P_{1}^{i *}=\left[\frac{1}{\mu \Omega_{1}^{i}+\lambda \frac{H_{\mathrm{SR}}^{i}}{H_{\mathrm{RD}}^{i}} \Omega_{2}^{i}}-\frac{1}{\gamma_{1}^{i}}\right]^{+} .
$$

By obtaining the optimal values of the transmitted powers $P_{1}^{i *}$ and $P_{2}^{i *}$, the dual function is now a function of $\mu$ and $\lambda$. In the next subsection, we use an algorithm named sub-gradient algorithm [12] that proceeds to the search of the optimum values of $\mu$ and $\lambda$ iteratively.

\subsubsection{Sub-gradient method to solve the dual problem}

With the obtained optimal values of primal variables $\left(P_{1}^{i *}, P_{2}^{i *}\right)$, the dual problem can be solved using the subgradient method [12-14]. In fact, our algorithm is based on the calculation of the Lagrangian multipliers $\lambda$ and $\mu$ in each iteration. The decision about the type of relaying mode over each sub-carrier is made using (10). The implementation procedures is described in the power allocation algorithm depicted in Algorithm 1.

The parameter $\delta^{(k)}$ appears in lines 14 and 15 of Algorithm 1, denoting the step size of the $k$ th iteration. This algorithm is well described in [12-14], where many types of step size can be used in the sub-gradient algorithm. In our model, we tried different step sizes and then used the best one in terms of best performance and less

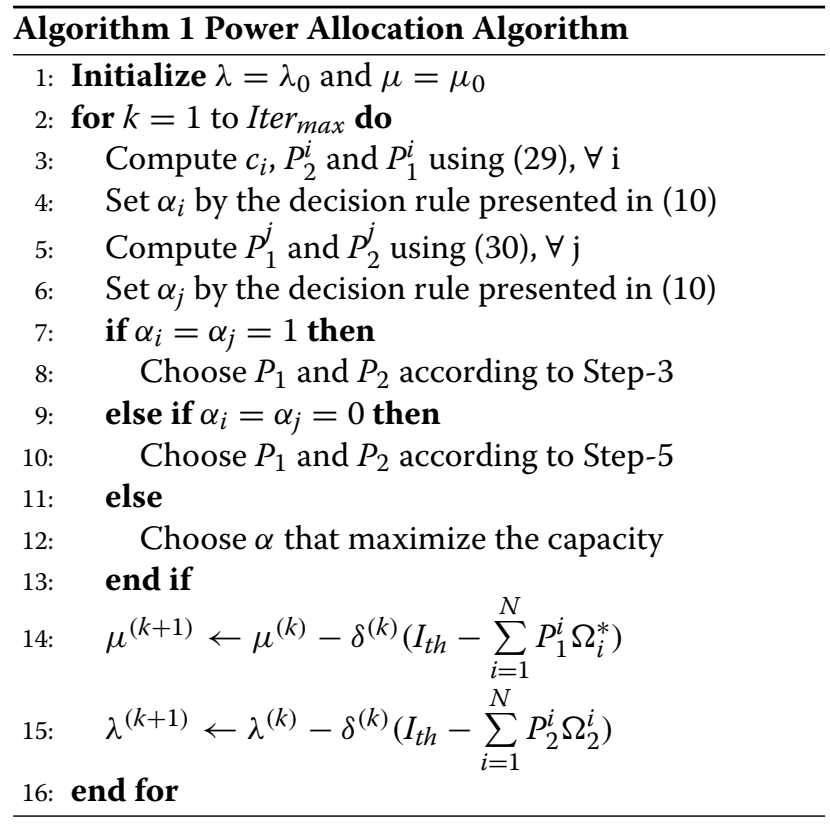

complexity. In the proposed scheme, the optimal power requires $\left(N^{2}\right)$ function evaluations for every sub-carrier to be matched in the second time slot. Therefore, the complexity of the proposed algorithm is $\mathcal{O}\left(T N^{2}\right)$, where $T$ is the number of iterations required for convergence. A comparison between the different schemes used in this paper is derived in Section 3.3.

\subsection{Simulation results}

The simulations are performed under the scenario given in Section 2.1. An OFDM system of $N$ sub-carriers $(N \in$ $\{16,32,64\})$ at the source and destination and one relay system is assumed. The values of $T_{\mathrm{s}}, \Delta f$, and $I_{\text {th }}$ are assumed to be $4 \mu \mathrm{s}, 0.3125 \mathrm{MHz}$, and $-20 \mathrm{dBm}$, respectively. The channel gains are outcomes of independent Rayleigh distributed random variables with mean equal to 1.

Figure 3 plots the average capacity using the different schemes (AAF, DAF, and ARP) vs. the SNR and using different values of total number of sub-carriers with $N=\{16,32,64\}$. It is shown that for low values of SNR $\left(\frac{1}{2 \sigma^{2}}<7\right)$ and for each value of $N$, the DAF relay decoding procedure is not perfect. Therefore, the AAF performs better than the DAF and provides higher capacity. However, at high SNR ( $>7)$ values, the behavior of the system become inverse to the previous situation. Here, decoding can be done 'perfectly', and the propagation of errors due to the amplification in AAF process has more chances to occur. Thus, in this SNR region, the performance achieved by the DAF mode is higher than that achieved by the AAF.

It can be also shown that the ARP relaying protocol achieves, for the different depicted values of SNR, the best 


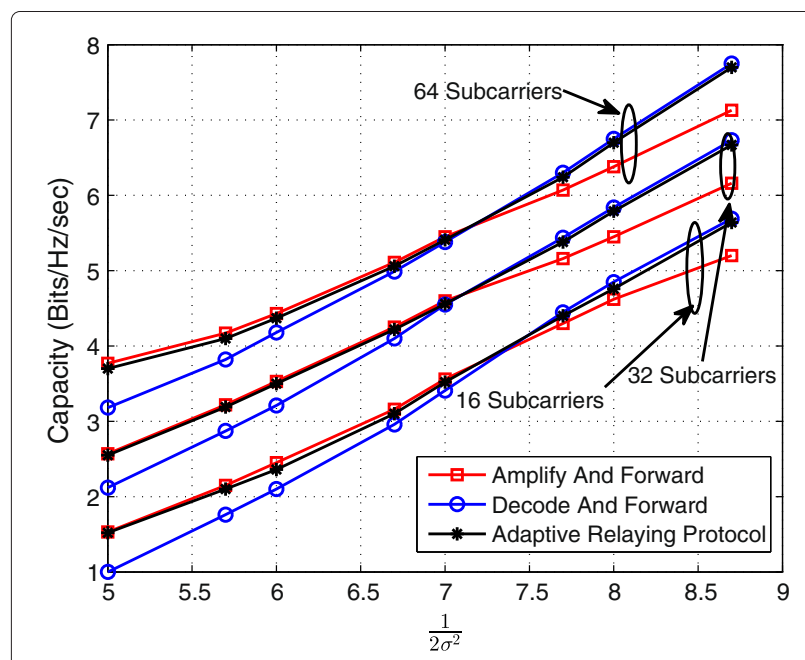

Figure 3 Effect of sub-carrier number on the capacity. Achieved capacity for different SNR values and different numbers of sub-carriers, $I_{\text {th }}=10^{-5} \mathrm{~W}$.

results. This can be explained by the fact that the ARP protocol is able to switch (in an adaptive way) from one relaying mode to another (AAF or DAF) using in each moment the relaying mode that achieves the best performance. In other words, the ARP tends to use the AAF relaying protocol for low values of SNR, and use the DAF for higher SNRs. Thus, ARP is able to take advantage of each relaying mode, depending on the SNR range. Figure 3 shows, finally, how the system capacity scales as function of the increase in the total number of carriers of the system.

Figure 4 depicts the average capacity using all the relaying schemes (AAF, DAF, and ARP) having two interference

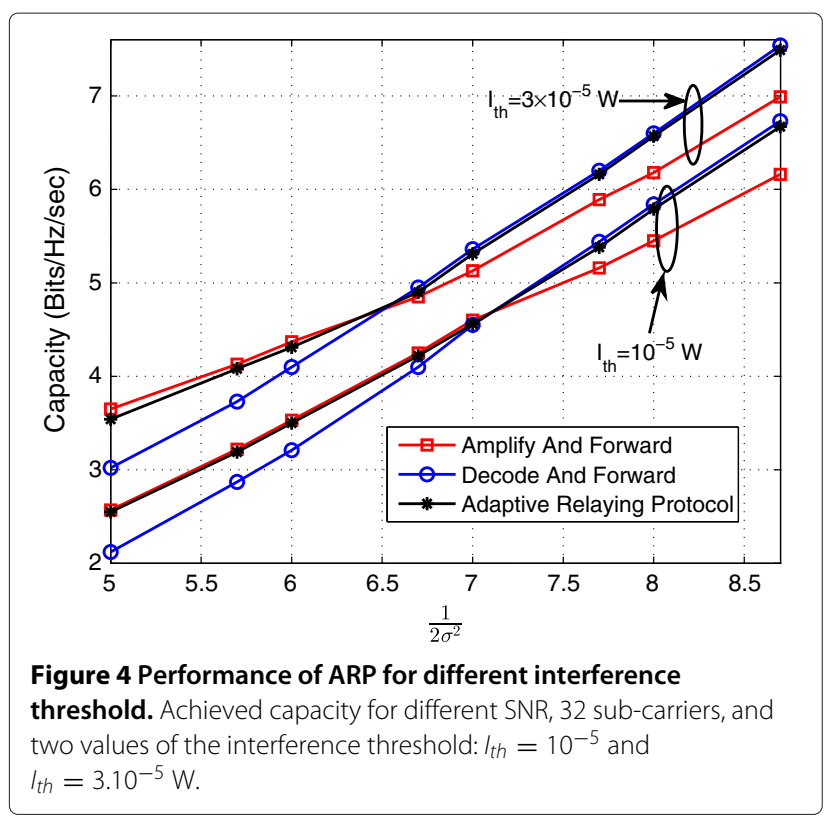

threshold values which are $10^{-5}$ and $3 \times 10^{-5} \mathrm{~W}$. It can be shown that the crossing point between the DAF and the AAF curves occurs at different SNR values when the system has different interference constraints. Note that for high values of interference threshold, the source and the relay will be able to transmit with more power than with small interference constraints $\left(I_{t h}\right.$ is small). This result implies that the decode procedure can be done correctly at the relay phase even for a high noise variance.

Figure 5 shows the capacity of the system versus the SNR having the relay system at different distances from the source. A general observation is that the ARP achieves higher capacity when relay is near the destination, then the performances decrease as soon as we have the relay at middle distance between the $\mathrm{S}$ and $\mathrm{D}$, and near to the source, respectively. It can be shown that the crossing point between the use of the AAF or the DAF appears at lower values of SNR if the relay system is located near the source. This can be explained by the fact that the relay receives data at high SNR in this case, which means that decoding can be done correctly and as such the relay switches to DAF mode. However, when the relay is near the destination, the intersection point appears at high SNR. In this case, the received signal at the relay has a low SNR, which favors AAF since AAF performs better than DAF for low and moderate values of SNR.

As a general observation from Figures 3, 4, and 5, it can be shown that the ARP scheme behavior always reaches the optimal scheme for different SNR values. However, the major limitation of the proposed scheme is its complexity. Thus, a new algorithm with much less complexity is required to make a step towards possible real implementation. Further, work should focus on the development of

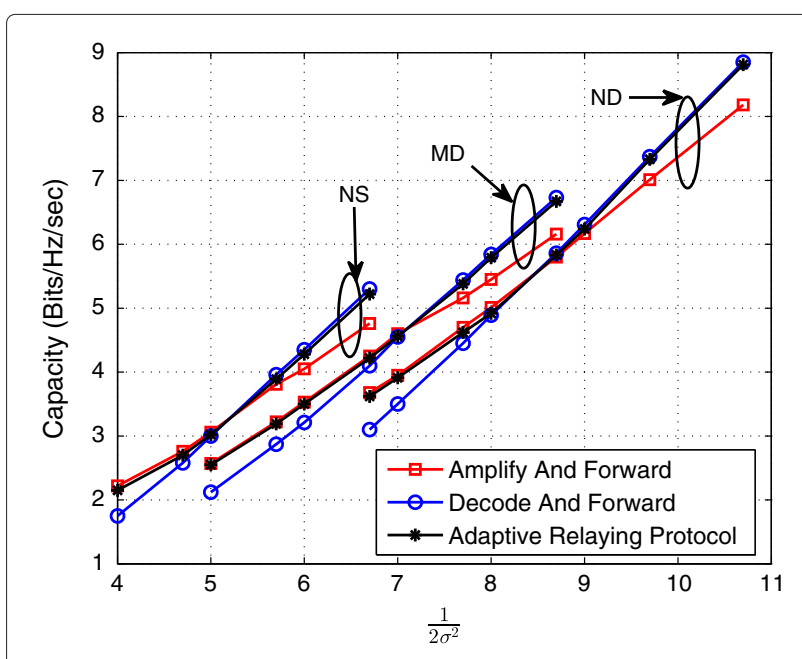

Figure 5 Performance of ARP for different positions of the relay. Achieved capacity for different SNR, different position of the relays, $I_{\text {th }}=10^{-5} \mathrm{~W}$, and 32 sub-carriers. NS, near source; MD, middle distance; ND, near destination. 
suboptimal algorithm that achieves a near optimal performance with affordable complexity of implementation.

Figure 6 shows capacity performance comparison using the matching and random pairing techniques for different values of SNR. In matching pairing technique, the same carrier $k$ is used in both time slots (in S-R and R-D links). However, with random pairing technique, the assigned carrier in the second time slot will be chosen randomly. It can be shown in this figure that higher capacity is achieved by matching carrier pairings than using the random assignment process of the carriers from S-R to R-D. It can be also observed that the ARP relaying technique achieves the best performances in both cases, matching and random pairing for different values of SNRs. We can conclude that using the matching pairing technique with ARP relaying strategy, higher capacity performance could be achieved for a wide range of SNR values.

The goal of Section 2 is to compare achieved performances between three relaying schemes, namely, the AAF, DAF, and ARP in a cognitive radio environment, using the matching sub-carrier technique. Given the superior performance of ARP, the next section focuses on the ARP scheme for a more complicated problem which includes the research of the best sub-carrier pairing to maximize the capacity under interference and power constraints.

\section{Sub-carrier pairing for adaptive relaying protocol}

As it was mentioned in the previous section, there are many types of pairing techniques to switch the subcarriers from the first link to the second link. It has been shown that the pairing strategy has an important impact on the resulting capacity. Therefore, in order to reach

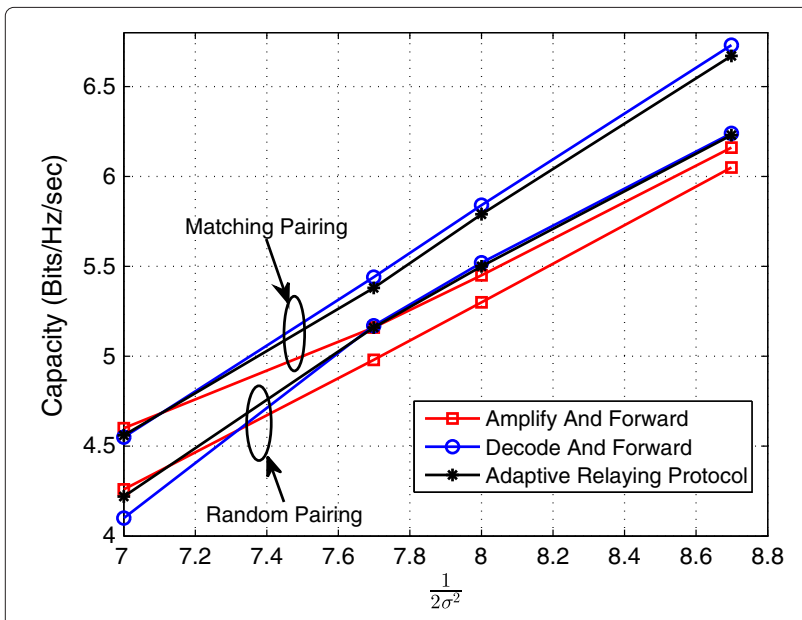

Figure 6 Impact of the sub-carrier pairing on the system capacity. Achieved capacity for different SNR, $I_{\text {th }}=10^{-5} \mathrm{~W}, 32$ sub-carriers, and two types of sub-carrier pairing: sub-carrier matching and random pairing. maximum capacity, with limited resources, we should carefully choose the pairing technique. One solution is to introduce the sub-carrier pairing in the final optimization problem in order to find the optimum pairing distribution that maximizes the capacity without increasing the complexity too much.

\subsection{System architecture}

The same OFDM cooperative system described in Section 2.1 is used in this section with some modification. In fact, a SU is present in the same coverage area of the PU and can communicate through the PU spectrum without causing harmful interference to the adjacent PUs. We assume the absence of a direct link between $S$ and $D$. Thus, the SU is reaching the destination using the ARP technique of relaying through one relay $\mathrm{R}$. It is assumed that the data is multiplexed into OFDM with several subcarriers whose total number is equal to $N$. Thus, the used spectrum by the CS is divided into $N$ sub-carriers, each having a $\Delta f$ bandwidth. Both $\mathrm{S}$ and $\mathrm{R}$ can transmit over the PU spectrum and interfere with its signal without exceeding the maximum interference power tolerated by PU, $I_{t h}$. As mentioned before, the source and the relay transmit in two different time slots in a way that the link $(\mathrm{S}-\mathrm{R})$ is active at the first time slot, while the link (R-D) is active in the second time slot.

Figure 7 illustrates the analyzed system. The main change in this model is at the relay side which has to distribute the sub-carriers to maximize the total rate. In fact, different pairs of sub-carrier $(k, l)$ that assure the transmission in both time slots are created, the $k$ th sub-carrier in the first time slot is paired with the $l$ th sub-carrier in the second time slot.

The interference calculations are the same as the ones done in Section 2.2, and the interference introduced by the $k$ th sub-carrier to $\mathrm{PU}, I_{k}\left(d_{k}, P_{k}\right)$, can as such be expressed as

$$
I_{k}\left(d_{k}, P_{k}\right) \triangleq P_{k} \Omega_{k}
$$

Also, the interference introduced by the PU is modeled as AWGN with a variance $J_{i}$. In what follows, the noise variance is denoted by $\sigma_{i}^{2}=\sigma^{2}$, and it is assumed to be the same for all sub-carriers and in both time slots.

\subsection{Problem formulation \\ 3.2.1 Total capacity}

The variables of the problem are defined as follows: $P_{1}^{k}$ $\left(P_{2}^{l}\right)$ is the power transmitted over the $k$ th $(l$ th $)$ sub-carrier in the S-R (R-D) link, respectively. The $k$ th (lth) subcarrier channel gain over the S-R (R-D) link is given by $H_{1}^{k}\left(H_{2}^{l}\right)$, respectively. Using the same notation used in the previous section for the transmitted and received signals 


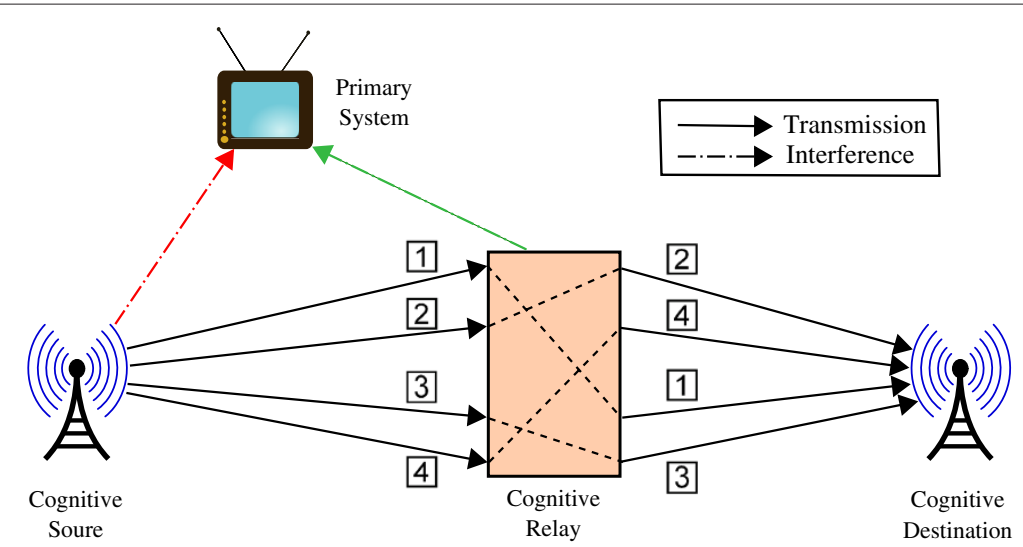

Figure 7 Cooperative relay cognitive radio network with sub-carrier pairing. Cooperative relay cognitive radio network with $N=4$. Dashed lines show the pairing over relay, and center lines represent the interference.

in the different system hops, we can show that the capacity in the first time slot can be written as follows:

$$
\mathcal{C}_{1, k}=\frac{1}{2} \log _{2}\left(1+P_{1}^{k} \gamma_{1}^{k}\right)
$$

However, in the second time slot, we get two possible formulas that depend on the type of forwarding technique used at the relay. Hence, if the sub-carriers pair $(k, l)$ is used for transmission, the achieved capacity in the second time slot is given by

$$
\mathcal{C}_{2}[k, l]=\left\{\begin{array}{cc}
\mathcal{C}_{\mathrm{DF}}[k, l]= & \frac{1}{2} \log _{2}\left(1+P_{2}^{l} \gamma_{2}^{l}\right) \\
& \text { if DAF is used } \\
\mathcal{C}_{\mathrm{AF}}[k, l]= & \frac{1}{2} \log _{2}\left(1+\frac{P_{1}^{k} P_{2}^{l} \gamma_{1}^{k} \gamma_{2}^{l}}{P_{2}^{l} \gamma_{2}^{l}+P_{1}^{k} \gamma_{1}^{k}+1}\right) \\
& \text { if AAF is used. }
\end{array}\right.
$$

Note that the same amplification factor defined in (8) is used by the relay in the AAF case. The variable $\gamma$ was given in Section 2.3 and is defined as

$$
\left\{\begin{array}{l}
\gamma_{1}^{k}=\frac{H_{1}^{k}}{\sigma^{2}} \\
\gamma_{2}^{l}=\frac{H_{2}^{l}}{\sigma^{2}}
\end{array}\right.
$$

For each channel couple $(k, l)$, the total rate is the minimum rate between both time slots. If the couple $(k, l)$ is using the DAF, the rate can be obtained by simply equalizing $\mathcal{C}_{1, k}$ to $\mathcal{C}_{D F}[k, l]$. This equality reduces the number of variables by making a relation between the power allocation in the first and second links as $P_{2}^{l}=\frac{\gamma_{1}^{k}}{\gamma_{2}^{l}} P_{1}^{k}$ for the channel couple $(k, l)$. However, if $(k, l)$ is operating under the AAF mode, the following inequality solves the problem, but does not reduce the number of variables

$$
P_{1}^{k} \gamma_{1}^{k}>\frac{P_{1}^{k} P_{2}^{l} \gamma_{1}^{k} \gamma_{2}^{l}}{P_{2}^{l} \gamma_{2}^{l}+P_{1}^{k} \gamma_{1}^{k}}
$$

As defined in (14), we can use the binary variable $\alpha$ to combined the two values of the capacity according to the forwarding technique. In fact, we know that $\alpha_{l}=1$ indicates that the $l$ th sub-carrier is operating on AAF, and $\alpha_{l}=0$ means that the $l$ th sub-carrier forwards by DAF. Furthermore, we introduce a new parameter $t_{k, l}$, to obtain the optimal possible combination $(k, l)$ in pairing at $\mathrm{R}$. In fact $t_{k, l}$ takes the values ' 0 ' and ' 1 '. The ' 1 ' means that the couple $(k, l)$ exists, and ' 0 ' in the case the couple $(k, l)$ does not exist. If we model $t_{k, l}$ by an $(N \times N)$ matrix $T$, the constraints on $t_{k, l}$ imply that the sum over each column of $T$ equals to 1 , and the sum over each row of $T$ equals also to 1 . The simplest choice of $T$ is the identity. In this case, $\mathrm{R}$ forwards the signal over the same received sub-carrier (see Section 2). To get a closed form of the total rate of the cognitive system, we introduce the previous changes to get the following expression:

$$
\mathcal{C}=\sum_{k=1}^{N} \sum_{l=1}^{N} t_{k, l}\left(\alpha_{l} \mathcal{C}_{\mathrm{AF}}[k, l]+\left(1-\alpha_{l}\right) \mathcal{C}_{\mathrm{DF}}[k, l]\right) .
$$

By writing the final expression of the total system capacity, a new constraint on the indicators appears. The first relates to the sub-carrier pairing constraint about $t_{k, l}$, and the second is about $\alpha_{l}$ :

$$
\begin{aligned}
\sum_{k=1}^{N} t_{k, l} & =1, \forall l ; \quad \sum_{l=1}^{N} t_{k, l}=1, \forall k \\
\alpha_{l} & =0 \text { or } 1, \forall l .
\end{aligned}
$$




\subsubsection{Optimization problem}

Our objective is to maximize the CR system throughput by optimizing the sub-carrier pairing and the power allocation at the source and the relay among assigned subcarrier pairs by taking care of the instantaneous interference introduced to PU which should be below a maximum limit. The interference constraints can be defined using the interference analysis developed in Section 2.2 to get the following equations:

$$
\begin{aligned}
& \sum_{k=1}^{N} P_{1}^{k} \Omega_{1}^{k} \leq I_{t h}, \\
& \sum_{l=1}^{N} P_{2}^{l} \Omega_{2}^{l} \leq I_{t h},
\end{aligned}
$$

where $\Omega_{1}^{k}$ and $\Omega_{2}^{l}$ are the interference factors in each slot.

Therefore, we can formulate the optimization problem as follows:

$$
\begin{aligned}
& \max _{P_{1}^{k}, P_{2}^{l}, \alpha_{l}, t_{k, l}} \mathcal{C} \\
& \text { s.t. }
\end{aligned}
$$

$-(\mathrm{C} 1$ : Source power constraint $)$

$$
\sum_{k=1}^{N} P_{1}^{k} \leq P_{\mathrm{S}}
$$

$-(\mathrm{C} 2$ : Relay power constraint)

$$
\sum_{k=1}^{N} P_{2}^{l} \leq P_{\mathrm{R}}
$$

$-(\mathrm{C} 3$ : First time slot interference)

$$
\sum_{k=1}^{N} P_{1}^{k} \Omega_{1}^{k} \leq I_{t h}
$$

$-($ C4: Second time slot interference)

$$
\sum_{l=1}^{N} P_{2}^{l} \Omega_{2}^{l} \leq I_{t h}
$$

$-(\mathrm{C} 5$ : Forwarding technique constraint)

$$
\alpha_{l} \in\{0,1\}, \forall l
$$

-(C6: Sub-carrier pairing constraint)

$$
\sum_{k=1}^{N} t_{k, l}=1, \forall l ; \quad \sum_{l=1}^{N} t_{k, l}=1, \forall k .
$$

$P_{\mathrm{S}}$ and $P_{\mathrm{R}}$ are the available power budgets in the source and the relay, respectively. The instantaneous fading gains are assumed to be perfectly known. The channel gains can be estimated using classical channel estimation techniques.

\subsection{Optimal power allocation}

Solving problem (39) with respect to the optimization variables $P_{1}^{k}, P_{2}^{l}$, and $t_{k, l}$ is a mixed binary integer programming problem. The problem in (39) is satisfying the time sharing condition presented in [30] for larger N. By consequence, the duality gap of the problem is negligible as the number of sub-carrier is sufficiently large (i.e.,
$N>8$ ) regardless of the convexity of the problem. By solving the dual problem, we get an asymptotically optimal solution [30]. To formulate and solve the dual problem, we need to find the Lagrangian of the primal problem which is given in (40).

$$
\begin{aligned}
\mathcal{L}= & \sum_{k=1}^{N} \sum_{l=1}^{N} \frac{1}{2} t_{k, l}\left[\alpha_{l} \log _{2}\left(1+\frac{P_{1}^{k} \gamma_{1}^{k} P_{2}^{l} \gamma_{2}^{l}}{P_{1}^{k} \gamma_{1}^{k}+P_{2}^{l} \gamma_{2}^{l}}\right)\right. \\
& \left.+\left(1-\alpha_{l}\right) \log _{2}\left(1+P_{1}^{k} \gamma_{1}^{k}\right)\right]+\beta\left(P_{\mathrm{S}}-\sum_{k=1}^{N} P_{1}^{k}\right) \\
& +\theta\left(P_{\mathrm{R}}-\sum_{l=1}^{N} P_{2}^{l}\right)+\lambda\left(I_{t h}-\sum_{k=1}^{N} P_{1}^{k} \Omega_{1}^{k}\right) \\
& +\mu\left(I_{t h}-\sum_{l=1}^{N} P_{2}^{l} \Omega_{2}^{l}\right) .
\end{aligned}
$$

The dual problem associated to the primal problem is given by

$$
\min _{\beta \geq 0 ; \theta \geq 0 ; \lambda \geq 0 ; \mu \geq 0} g(\beta, \theta, \lambda, \mu),
$$

where $\beta$ and $\theta$ are the Lagrangian multipliers (dual variables) related to the power constraints at the source and the relay, and $\lambda$ and $\mu$ represent the dual variables associated to the interference constraints. The dual function $g$ is defined as

$$
\begin{gathered}
g(\beta, \theta, \lambda, \mu)=\max _{P_{1}^{k}>0 . P_{2}^{l}>0, \alpha_{l}, t_{k, l}} \mathcal{L} \\
\text { s.t. } \quad(\mathrm{C} 5),(\mathrm{C} 6) .
\end{gathered}
$$

We can rewrite the dual function from (42) as follows:

$$
\begin{gathered}
g(\beta, \theta, \lambda, \mu)=\max _{P_{1}^{k}>0, P_{2}^{l}>0, \alpha_{l}, t_{k, l}} \\
\sum_{k=1}^{N} \sum_{l=1}^{N} t_{k, l}\left(\alpha_{l} \mathcal{D}_{\mathrm{AF}}\left(P_{1}^{k}, P_{2}^{l}\right)+\left(1-\alpha_{l}\right) \mathcal{D}_{\mathrm{DF}}\left(P_{1}^{k}\right)\right) \\
+\beta P_{\mathrm{S}}+\theta P_{\mathrm{R}}+I_{t h}(\lambda+\mu) \\
\text { s.t. } \quad(\mathrm{C} 5),(\mathrm{C} 6),
\end{gathered}
$$

where we introduced two new functions in the dual function to simplify the computation, given by

$$
\begin{aligned}
\mathcal{D}_{\mathrm{AF}}\left(P_{1}^{k}, P_{2}^{l}\right)= & \frac{1}{2} \log _{2}\left(1+\frac{P_{1}^{k} \gamma_{1}^{k} P_{2}^{l} \gamma_{2}^{l}}{P_{1}^{k} \gamma_{1}^{k}+P_{2}^{l} \gamma_{2}^{l}}\right) \\
& -\beta P_{1}^{k}-\theta P_{2}^{l}-\lambda P_{1}^{k} \Omega_{1}^{k}-\mu P_{2}^{l} \Omega_{2}^{l},
\end{aligned}
$$

and

$$
\begin{aligned}
\mathcal{D}_{\mathrm{DF}}\left(P_{1}^{k}, l\right)= & \frac{1}{2} \log _{2}\left(1+P_{1}^{k} \gamma_{1}^{k}\right)-\beta P_{1}^{k}-\theta \frac{P_{1}^{k} \gamma_{1}^{k}}{\gamma_{2}^{l}} \\
& -\lambda P_{1}^{k} \Omega_{1}^{k}-\mu \frac{P_{1}^{k} \gamma_{1}^{k}}{\gamma_{2}^{l}} \Omega_{2}^{l} .
\end{aligned}
$$


Moreover, for a given values of the different dual variables, we get two cases depending on the value of the variable $\alpha_{l}$ :

- Case 1 : The pair $(k, l)$ is used for amplify and forward, i.e., $\alpha_{l}=1$. Assume $(k, l)$ to be a valid sub-carrier pair; the optimal power allocation can be evaluated by solving the following sub-problem for every $(k, l)$ assignment:

$$
\max _{P_{1}^{k}, P_{2}^{l}} \mathcal{D}_{\mathrm{AF}}\left(P_{1}^{k}, P_{2}^{l}\right) \quad \text { s.t. } \quad P_{1}^{k} \geq 0, P_{2}^{l} \geq 0 .
$$

Hence, we obtain the optimal power by equating

$$
\frac{\partial \mathcal{D}_{\mathrm{AF}}\left(P_{1}^{k}, P_{2}^{l}\right)}{\partial P_{1}^{k}}=\frac{\partial \mathcal{D}_{\mathrm{AF}}\left(P_{1}^{k}, P_{2}^{l}\right)}{\partial P_{2}^{l}}=0 .
$$

The optimal power in (46) can be expressed as follows:

$$
\left\{\begin{aligned}
P_{1}^{k *} & =\left[\frac{\gamma_{1}^{k}}{\left(\gamma_{1}^{k}+c_{k, l} \gamma_{2}^{l}\right) c_{k, l}\left(\theta+\mu \Omega_{2}^{l}\right)}-\frac{1}{\gamma_{1}^{k}}-\frac{1}{c_{k, l} \gamma_{2}^{l}}\right]^{+} \\
P_{2}^{l *} & =c_{k, l} P_{1}^{k},
\end{aligned}\right.
$$

where $c_{k, l}=\sqrt{\frac{\gamma_{1}^{k}\left(\beta+\lambda \Omega_{1}^{k}\right)}{\gamma_{2}^{l}\left(\theta+\mu \Omega_{2}^{l}\right)}}$. Hence, the power variable in (43) can be eliminated by substituting the optimal power allocation found in (48). Then, the dual function can be easily found by searching the optimal pair $(k, l)$ that maximizes the dual function.

- Case 2: The pair $(k, l)$ is used for decode and forward, i.e., $\alpha_{l}=0$. In this case, we assume that the pair $(k, l)$ is a valid pair that forwards by DAF technique. The following problem should be solved for each valid pair

$$
\max _{P_{1}^{k}, P_{2}^{l}} \mathcal{D}_{\mathrm{DF}}\left(P_{1}^{k}, l\right) \quad \text { s.t. } \quad P_{1}^{k} \geq 0 .
$$

By differentiating the previous function over $P_{1}$, we obtain the optimal power allocation in this case:

$$
\left\{\begin{array}{l}
P_{1}^{k *}=\left[\frac{1}{\beta+\theta \frac{\gamma_{1}^{k}}{\gamma_{2}^{l}}+\lambda \Omega_{1}^{k}+\mu \frac{\gamma_{1}^{k}}{\gamma_{2}^{l}} \Omega_{2}^{l}}-\frac{1}{\gamma_{1}^{k}}\right]^{+} \\
P_{2}^{l *}=\frac{\gamma_{1}^{k}}{\gamma_{2}^{l}} P_{1}^{k} .
\end{array}\right.
$$

Like case 1 , we substituted the power variable by its optimal value to get a new problem without power parameter. Therefore, the best pair $(k, l)$ is chosen so it maximizes the dual function.
At this stage, we get the power allocation and subcarrier pairing in function of the dual variables so that the dual function can be written as follows:

$$
\begin{gathered}
g(\beta, \theta, \lambda, \mu)= \\
\max _{t_{k, l}} \sum_{k=1}^{N} \sum_{l=1}^{N} t_{k, l}\left(\alpha_{l} \mathcal{D}_{\mathrm{AF}}\left(P_{1}^{k *}, P_{2}^{l *}\right)+\left(1-\alpha_{l}\right) \mathcal{D}_{\mathrm{DF}}\left(P_{1}^{k *}\right)\right) \\
+\beta P_{\mathrm{S}}+\theta P_{\mathrm{R}}+I_{t h}(\lambda+\mu) \\
\text { s.t. } \quad(\mathrm{C} 6) .
\end{gathered}
$$

The problem in (51) is a linear optimization problem which can be simply solved. The sub-gradient method can be used to solve the dual problem with guaranteed convergence. At this state, we get all the optimal solution, i.e., $P_{1,2}^{*}, t_{k, l}^{*}$ and $\alpha_{l}$ of the dual function for a given dual points $\beta, \theta, \lambda$, and $\mu$. The dual variables at the $(i+1)$ th iteration are then updated as

$$
\begin{aligned}
& \beta^{(i+1)}=\beta^{(i)}-\delta^{(i)}\left(P_{\mathrm{S}}-\sum_{k=1}^{N} P_{1}^{k *}\right) \\
& \theta^{(i+1)}=\theta^{(i)}-\delta^{(i)}\left(P_{\mathrm{R}}-\sum_{l=1}^{N} P_{2}^{l *}\right) \\
& \lambda^{(i+1)}=\lambda^{(i)}-\delta^{(i)}\left(I_{t h}-\sum_{k=1}^{N} P_{1}^{k *} \Omega_{1}^{k}\right) \\
& \mu^{(i+1)}=\mu^{(i)}-\delta^{(i)}\left(I_{\text {th }}-\sum_{l=1}^{N} P_{2}^{l *} \Omega_{2}^{l}\right),
\end{aligned}
$$

where $\delta^{(i)}$ is the step size that can be updated according to the non-summable diminishing step size policy [12-14].

As shown before, the complexity to get the optimal power in the source side is $N^{2}$ for every sub-carrier. The pairing scheme requires $N$ more operation to get the total power allocation. Therefore, the complexity of the proposed algorithm in the pairing mode is $\mathcal{O}\left(T N^{3}\right)$ where $T$ is the number of iteration required for convergence. Table 1 summarizes the complexity of the different schemes used in this paper.

\subsection{Simulation results}

According to the scenario given in Section 3.1, a multicarrier system of $N=32$ sub-carrier and single relay is assumed. The values of the symbol duration $T_{\mathrm{s}}, \Delta f$, and

Table 1 Complexity of the used algorithms

\begin{tabular}{lc}
\hline Scheme & Complexity \\
\hline Sub-gradient algorithm & $\mathcal{O}(T N)$ \\
ARP with sub-carrier mathcing & $\mathcal{O}\left(T N^{2}\right)$ \\
ARP with sub-carrier pairing & $\mathcal{O}\left(T N^{3}\right)$ \\
\hline
\end{tabular}


$\sigma^{2}$ are assumed to be $4 \mu \mathrm{s}, 0.3125 \mathrm{MHz}$, and $10^{-7}$, respectively, as it was the case in Section 2.5. The channel gains are outcomes of independent Rayleigh distributed random variables with a mean equal to 1 .

Figure 8 shows the achieved capacity for different interference and power constraints using the adaptive relaying protocol. From the figure, it can be observed that the capacity increases as the other constraints increase. However, by fixing one of the constraints, the capacity does not change and becomes constant when some value of the other constraint is reached; this can be justified by the fact that the power allocation reaches its maximum value allowed by the changeable constraint and can not move beyond this constraint. An example of this case is clearly shown in Figure 9, where the evolution of the average capacity versus the interference threshold for two values of the power constraint is drawn. Note that for a fixed power constraint, the system capacity becomes constant because the interference introduced to the PU using the fixed power budget is less than the interference threshold. On the other hand, the figure shows us also that the optimal pairing strategy has the best performance compared to the matched pairing strategy studied in the previous chapter or any random pairing scheme. In fact, in the optimal pairing strategy, the algorithm chooses the best combination of sub-carrier pairs to be used while relaying which is, as expected, better than using the same sub-carrier for relaying or using a randomly picked set of sub-carrier couples.

The system used above assumes that the relay is located in the middle between the source and the destination because the channel gains have the same power. The position of the relay can be changed by modifying the channel power (CS to $R$ ) and (R to $D$ ). The results in Figure 10 confirm that the position of the relay has an impact on the

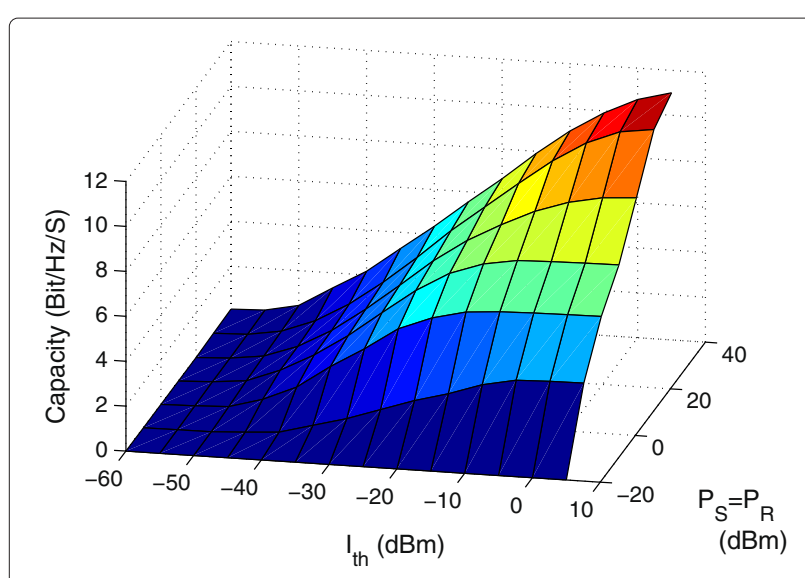

Figure 8 Performance of ARP with sub-carrier pairing. Achieved capacity as function of the allowed interference threshold and power budget constraints.

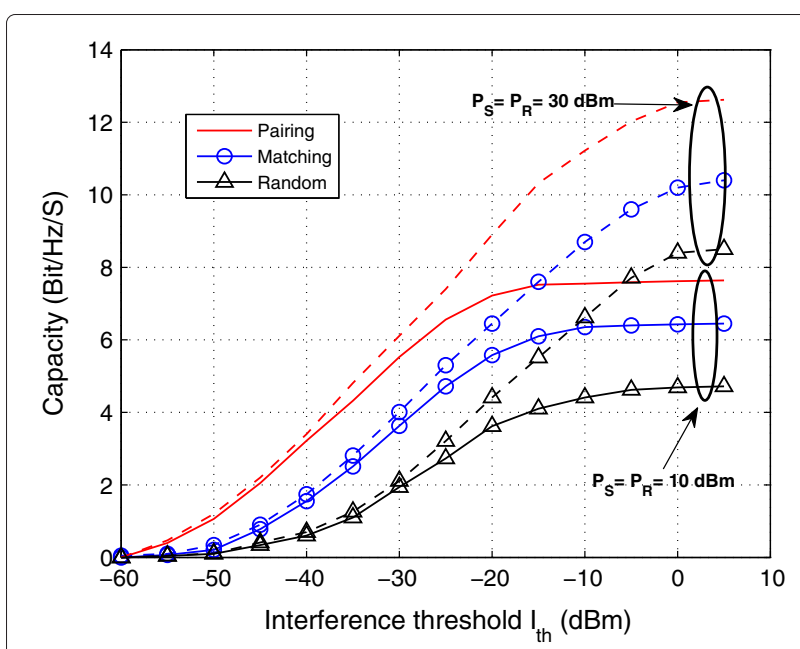

Figure 9 Effect of interference threshold and power budget on the ARP. Achieved capacity vs allowed interference threshold (Solid lines for $P_{\mathrm{S}}=P_{\mathrm{R}}=10 \mathrm{dBm}$ and dashed lines for $P_{\mathrm{S}}=P_{\mathrm{R}}=30 \mathrm{dBm}$ ).

system capacity. From this figure, it is shown that the best performance appears when the relay is near the source and the worst case when the relay is near the destination. Indeed, the relay receives a signal with high SNR when it is near the source. As such what ever the deployed forwarding technique (DAF or AAF), the relay transmits a signal with good quality, very near to the original signal for the DAF case and without an important noise amplification for the AAF case. However, as the relay moves away from the source, the quality of the received signal at $R$ degrades, and the processing becomes more difficult which affects

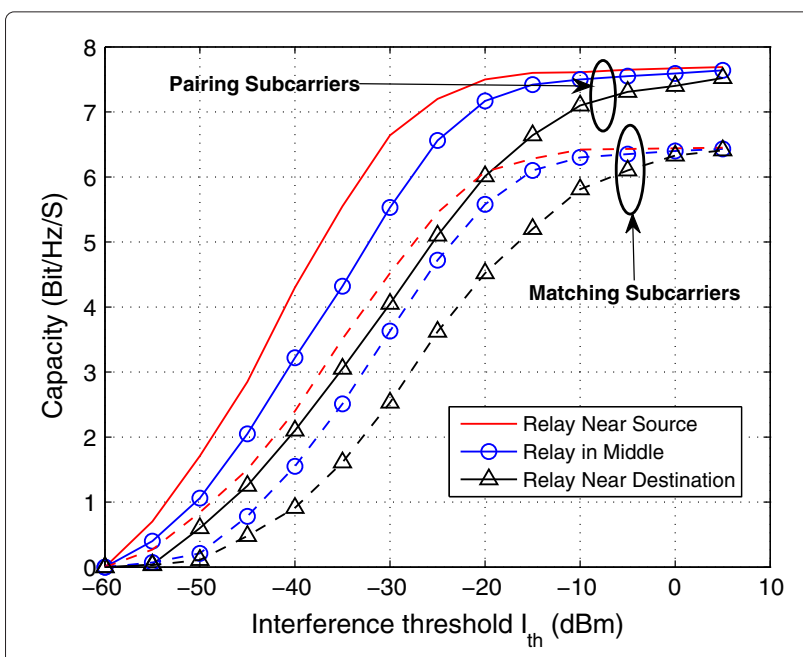

Figure 10 Performance of ARP with pairing for different positions of relay. System capacity for different values of interference threshold, different positions of the relay, and $P_{S}=P_{R}=10 \mathrm{dBm}$. Solid lines represent the pairing sub-carrier technique, and the dashed lines, the matching technique. 
the capacity of the system and decreases it. Therefore, the worst case appears when the relay is near the destination since the DAF will cause error propagation, while AAF will cause an important noise amplification because of the low received SNR. Moreover, the curves in this figure confirm the efficiency of the proposed scheme of pairing. Note that whatever the position of the relay, the system reaches the same maximum capacity allowed by the power budget for high interference threshold. This issue can be explained by the fact that for high interference threshold, the received SNR at the relay is very high even if the relay is far from the source. Therefore, the capacity increases as the interference threshold increases until it saturates when the power reaches its maximum value.

\section{Conclusion}

In this paper, we considered a near optimal power allocation algorithm for an OFDM-based system with adaptive relaying protocol using a single relay. In the first part, the problem is solved for the simple case of subcarrier matching to compare the performance of the ARP scheme to the classical AAF and DAF techniques. However, in the second part, the goal was to maximize the capacity by jointly optimizing the sub-carrier pairing, the power allocation, and the relaying technique (AAF or DAF). In our framework, we assumed a limited power budget at each transmitter, and because it is a cognitive scenario, the introduced interference to the primary user was required not to exceed a predetermined tolerated threshold. The problem was formulated with the different constraints as a mixed integer programming problem. We used the dual method to solve the optimization problem iteratively using the sub-gradient algorithm.

Some selected simulation results confirmed the efficiency of the proposed relaying scenario (ARP), which offers better performance in comparison to the AAF and DAF techniques. These results showed also that the performance has a considerable dependence on the adopted sub-carrier pairing techniques at the relay. The simulation results showed finally the effects of the interference threshold tolerated by the PU and the impact of the power budget set at the transmitters.

\section{Endnote}

${ }^{a}$ This formula assumed a perfect knowledge of the CSI. The outdated CSI scenario is studied in [24], when the average interference is expressed in terms of the interference factor $\Omega^{i}$, the channel gain $H^{i}$, and the correlation coefficient $\rho$ ([24], Eq. (16)). This case can be integrated in our model by changing the interference factor by the new outdated CSI factor.

\section{Competing interests}

The authors declare that they have no competing interests.

\section{Acknowledgements}

This work was supported in part by King Abdullah University of Science and Technology (KAUST), the European project ACROPOLIS-NoE (ICT-2009.1.1), the COST Action IC0902, and the NPRP grant N 5-250-2-087 from the Qatar National Research Fund (a member of Qatar Foundation). The statements made herein are solely the responsibility of the authors. This work is an expanded version of work presented at the International Conference on Cognitive Radio Oriented Wireless Networks (CROWNCOM 2012), Stockholm, Sweden, June 2012.

\section{Author details}

${ }^{1}$ Computer, Electrical, and Mathematical Sciences and Engineering (CEMSE) Division, King Abdullah University of Science and Technology (KAUST), Thuwal, Makkah Province, 23955-6900 Kingdom of Saudi Arabia. ${ }^{2}$ SUPELEC, Campus of Rennes, Avenue de la Boulaie CS 47601, F-35576 Cesson-Sévigné Cedex, France. ${ }^{3}$ Electrical Engineering Department, IUG University, P.O. Box 108, Gaza, Palestine.

Received: 11 May 2013 Accepted: 21 October 2013

Published: 9 November 2013

\section{References}

1. S Haykin, Cognitive radio: Brain-empowered wireless communications. IEEE J. Selected Areas Commun. 23(2), 201-220 (2005)

2. G Bansal, M Hossain, V Bhargava, Optimal and suboptimal power allocation schemes for OFDM-based cognitive radio systems. IEEE Trans. Wireless Commun. 7(11), 4710-4718 (2008)

3. M Shaat, F Bader, Computationally efficient power allocation algorithm in multicarrier-based cognitive radio networks OFDM and FBMC systems. EURASIP J. Adv. Signal Process. 2010, 528378 (2010)

4. $Y$ Zhang, C Leung, Resource allocation in an OFDM-based cognitive radio system. IEEE Trans. Commun. 57(7), 1928-1931 (2009)

5. $Y$ Jing, $\mathrm{H}$ Jafarkhani, Single and multiple relay selection schemes and their diversity orders, in Proceedings of the IEEE International Conference on Communications Workshops, (ICCW 2008), Beijing, China, 19-23 May 2008

6. Y Li, B Vucetic, On the performance of a simple adaptive relaying protocol for wireless relay networks, in Proceedings of the IEEE Vehicular Technology Conference, (VTC 2008-Spring), Singapore, 11-14 May 2008

7. A Khan, V Kühn, Power optimization in adaptive relay networks, in Proceedings of the IEEE Global Telecommunications Conference (GLOBECOM 2010), Miami, FL, USA, 6-10 Dec 2010

8. M Shaat, F Bader, Optimal power allocation algorithm for OFDM-based decode-and-forward dual-hop cognitive systems. Budapest, Hungary, 15-18 May 2011

9. V Asghari, S Aissa, Performance of cooperative spectrum-sharing with amplify-and-forward relaying. IEEE Trans. Wireless Commun. 11, 1295-1300 (2012)

10. Y Han, A Pandharipande, S Ting, Cooperative decode-and-forward relaying for secondary spectrum access. IEEE Trans. Wireless Commun. 8, 4945-4950 (2009)

11. H Soury, F Bader, M Shaat, MS Alouini, Near optimal power allocation algorithm for OFDM-based cognitive using adaptive relaying strategy, in Proceedings of the IEEE International Conference on Cognitive Radio Oriented Wireless Networks (CROWNCOM 2012), Sweden, 18-20 June 2012

12. NZ Shor, KC Kiwiel, A Ruszcayǹski, Minimization Methods for Non-differentiable Functions (Springer, NY, USA, 1985)

13. M Akgül, Topics in relaxation and ellipsoidal methods, in Research Notes In Mathematics Series, vol. 97, (Pitman Advanced Pub. Program, Boston, USA, 1984)

14. DP Bertsekas, Nonlinear Programming, 2nd edn, (Athena Scientific, Nashua, USA, 1999)

15. Y Zou, YD Yao, B Zheng, Cooperative relay techniques for cognitive radio systems: spectrum sensing and secondary user transmissions. IEEE Commun. Mag. 50(4), 98-103 (2012)

16. Y Zou, YD Yao, B Zheng, Cognitive transmissions with multiple relays in cognitive radio networks. IEEE Trans. Wireless Commun. 10(2), 648-659 (2011) 
17. M Shaat, F Bader, Asymptotically optimal resource allocation in OFDM-based cognitive networks with multiple relays. IEEE Trans. Wireless Commun. 11(3), 892-897 (2012)

18. $\mathrm{TNg}, \mathrm{W} \mathrm{Yu}$, Joint optimization of relay strategies and resource allocations in cooperative cellular networks. IEEE J. Selected Areas Commun. 25(2), 328-339 (2007)

19. G Sidhu, F Gao, W Wang, W Chen, Resource allocation in relay-aided OFDM cognitive radio networks. IEEE Trans. Vehicular Technol. 62(8), 3700-3710 (2013)

20. D Bharadia, G Bansal, P Kaligineedi, V Bhargava, Relay and power allocation schemes for OFDM-based cognitive radio systems. IEEE Trans. Wireless Commun. 10(9), 2812-2817 (2011)

21. W Wang, S Yang, L Gao, Comparison of schemes for joint subcarrier matching and power allocation in OFDM decode-and-forward relay system, in Proceedings of the IEEE International Conference on Communications (ICC), Beijing, China, 19-23 May 2008

22. CN Hsu, HJ Su, PH Lin, Joint subcarrier and power allocation for OFDM transmission with decode-and-forward relaying. IEEE Trans. Signal Process. 59, 399-414 (2011)

23. TWeiss, J Hillenbrand, A Krohn, F Jondral, Mutual interference in OFDM-based spectrum pooling systems, in Proceedings of the IEEE Vehicular Technology Conference, (VTC'O4-Spring), Milan, Italy, 17-19 May 2004

24. S Biyanwilage, U Gunawardana, R Liyanapathirana, New power allocation methods for AF relay assisted OFDM cognitive radio networks with outdated CSI, in Proceedings of the IEEE International Symposium on Communications and Information Technologies (ISCIT), Australia, 2-5 Oct 2012

25. TRiihonen, R Wichman, S Werner, Evaluation of OFDM(A) relaying protocols: capacity analysis in infrastructure framework. IEEE Trans. Vehicular Technol. 61, 360-374 (2012)

26. W Dang, M Tao, H Mu, J Huang, Subcarrier-pair based resource allocation for cooperative multi-relay OFDM systems. IEEE Trans. Wireless Commun. 9(5), 1640-1649 (2010)

27. J Tang, $X$ Zhang, Cross-layer resource allocation over wireless relay networks for quality of service provisioning. IEEE J. Selected Areas Commun. 25(4), 645-656 (2007)

28. W Yue, B Zheng, Q Meng, Optimal power allocation for cognitive relay networks, in Proceedings of the International Conference on Wireless Communications Signal Processing (WCSP 2009), Nanjing, China, 25-27 June 2009

29. R Zhang, S Cui, YC Liang, On Ergodic sum capacity of fading cognitive multiple-access and broadcast channels. IEEE Trans. Inf. Theory. 55(11), 5161-5178 (2009)

30. W Yu, R Lui, Dual methods for nonconvex spectrum optimization of multicarrier systems. IEEE Trans. Commun. 54(7), 1310-1322 (2006)

doi:10.1186/1687-1499-2013-259

Cite this article as: Soury et al.: Joint sub-carrier pairing and resource allocation for cognitive networks with adaptive relaying. EURASIP Journal on Wireless Communications and Networking 2013 2013:259.

\section{Submit your manuscript to a SpringerOpen ${ }^{\circ}$ journal and benefit from:}

- Convenient online submission

- Rigorous peer review

- Immediate publication on acceptance

- Open access: articles freely available online

- High visibility within the field

- Retaining the copyright to your article

Submit your next manuscript at $\boldsymbol{\triangleright}$ springeropen.com 